\title{
Symmetry of disciform scars in bilateral age-related macular degeneration
}

Department of Clinical Ophthalmology, Moorfields Eye Hospital, London EC1V M J Lavin

Hacettepe Medical Centre, Sihhiye, Ankara, Turkey

B Eldem

Moorfields Eye Hospital Z J Gregor

Correspondence to:

Mr Z J Gregor, Moorfields Eye Hospital, City Road, London ECIV 2PD.

Accepted for publication 21 September 1990

\author{
Michael J Lavin, Bora Eldem, Zdenek J Gregor
}

\begin{abstract}
The size of the final macular scar in subretinal neovascularisation (SRNV) is one of the most important determinants of final visual function in patients with subfoveal disease. We studied patients with bilateral macular scars from agerelated subretinal neovascular membranes retrospectively in order to determine whether or not fellow eyes behave similarly. We found a significant correlation between eyes in terms of final scar size $(r=0.50, p<0.01)$. We found that $50 \%$ of fellow eyes with large macular scars $\left(>3 \times 10^{6} \mu \mathrm{m}^{2}\right)$ had similar sized lesions, while only $16 \%$ of fellow eyes with small macular scars $\left(<0.5 \times 10^{6} \mu \mathrm{m}^{2}\right)$ had large scars $(\mathbf{p}<0.01)$. We discuss the significance of these findings in relation to the pathogenesis of subretinal neovascular membranes, and their implications for treatment.
\end{abstract}

Age-related macular degeneration (AMD) is the major cause of visual loss in the elderly.' Approximately $10 \%$ of patients with AMD suffer subretinal neovascular membrane (SRNVM) formation and its complications, though this is responsible for most of the legal blindness in this disease. ${ }^{2}$ When eyes with similar visual acuities are compared, patients with SRNVMs have much poorer visual function than those with other forms of AMD. ${ }^{3}$ Since the quality of paracentral visual function is related to the retinal distance from the fovea, ${ }^{4}$ and exudative AMD has a high incidence of bilaterality, ${ }^{5}$ we wished to know whether or not the retinal scar size resulting from exudative AMD was similar between eyes in bilateral cases. This could provide useful information on the likely visual prognosis in the good eye in individual patients and have important implications for possible treatment.

There is evidence that features of nonexudative AMD are symmetrical ${ }^{6}$ and that the type and extent of drusen formation is highly correlated between eyes in patients with nonexudative AMD. ^ Although symmetry has been demonstrated for one complication of AMD, namely tears of the retinal pigment epithelium, ${ }^{8}$ it is not known whether or not the high degree of concordance seen in drusen is reflected in the behaviour of other exudative complications of AMD. Since SRNVMs form the bulk of cases of exudative AMD, ${ }^{9}$ we limited our study to cases with documented SRNVM formation.

While these SRNVMs may under some circumstances be amenable to laser treatment, ${ }^{10}$ " a large number of SRNVMs are not treatable by conventional means. ${ }^{10}$ Since the final scar size, in

^Barondes MJ, Pauleikhoff D, Chisholm IH, Minassian D, Bird AC, unpublished. the presence of foveal involvement, is a major determinant of subsequent paracentral visual function, ${ }^{12}$ some workers have suggested that ablation of subfoveal membranes may be justified in order to limit the final scar size and reduce the resultant scotoma ${ }^{1314}$ when the fellow eye has a poor outcome from AMD. The final scar size resulting from SRNVMs can be highly variable. We wished to know whether or not it would be possible to identify factors indicating a high risk of large scars' forming.

\section{Material and methods}

We performed a retrospective study of patients with bilateral disciform macular scars resulting from age-related macular degeneration. Consecutive patients with bilateral disciform macular scars were identified from a database of patients attending the Retinal Diagnostic Department at Moorfields Eye Hospital. Stereoscopic colour photographs and fluorescein angiograms were examined in order to determine eligibility. Patients were included in the study only if their bilateral macular scarring was the result of subretinal neovascular membrane formation secondary to age-related macular degeneration. Patients with pigment epithelial detachment were included if there was clear evidence of subretinal neovascularisation. Patients with atrophic macular lesions, or geographic atrophy, were excluded. Patients who had had laser photocoagulation to either eye at any time were excluded. A minimum duration of subretinal neovascularisation of six months was required.

Stereo colour photographs and fluorescein angiograms were examined independently by two observers (ZJG and MJL), and scar size was measured by a standardised photographic slide overlay. ${ }^{5}$ The scar size was ascertained from colour photographs and fluorescein angiograms by measuring from the edges of subretinal fibrovascular tissue. The extent of subretinal fluid or blood was not included in these measurements. The area of fibrosis apparent on the colour photographs was usually less than the area of neovascularisation visible angiographically, and where a difference existed the larger measurement was used. Area approximations were arrived at by multiplying the length of the longest axis of the scar wth the scar diameter perpendicular to it.

A total of 100 patients with bilateral established macular scarring resulting from subretinal neovascularisation in age-related macular degeneration were identified. Of these, 19 patients were not included owing to inadequate follow-up, leaving a study population of 81 patients.

Interobserver agreement error was tested with 


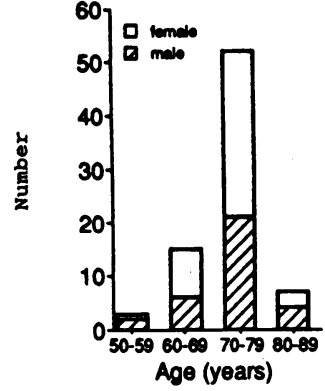

Figure 1 Age and sex distribution of patients. Accurate age data not available for 4 patients. a random series of 10 eyes. Measurements were similar between two observers for 10 eyes, with no error exceeding $10 \%$.

Results

The patients ranged in age from 54 to 86 years, with a mean of $69 \cdot 7$ (SD 17.0) years (Fig 1). Patients with large scar sizes $\left(>3 \times 10^{6} \mu \mathrm{m}^{2}\right)$ tended to be older than those with small scars, but this difference was not statistically significant. Analysis of covariance found age was not a variable associated with scar size.

The distribution of scar size in our patients (Fig 2) appeared to have two peaks: an initial peak of under $0.5 \times 10^{6} \mu \mathrm{m}^{2}$ in area, accounted for $50 / 162(30.9 \%)$ of eyes. While 28 eyes $(17 \cdot 2 \%)$ had scar sizes ranging between 0.5 and $0.99 \times 10^{6} \mu \mathrm{m}^{2}$, only small percentages of eyes fell in the intervening ranges of 1 to $3 \times 10^{6} \mu \mathrm{m}^{2}$ in area. A second peak was found in the 3 to $4 \times$ $10^{6} \mu \mathrm{m}^{2}$ range which accounted for $42 / 162$ $(25 \cdot 9 \%)$ of eyes (Fig 2).

Scars of less than $0.5 \times 10^{6} \mu \mathrm{m}^{2}$ in area were found in 50 eyes of 36 patients. The distribution of scar sizes in the fellow eye of patients with one scar measuring less than $0.5 \times 10^{6} \mu \mathrm{m}^{2}$ is charted in Figure 3. In $38 \cdot 8 \%(14 / 36)$ of fellow eyes, scars

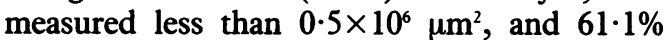
were less than $1 \times 10^{6} \mu \mathrm{m}^{2}$ in area. Only $16.7 \%$ (6/36) had large scar sizes $\left(>3 \times 10^{6} \mu \mathrm{m}^{2}\right)$.

Scars measuring $3 \times 10^{6} \mu \mathrm{m}^{2}$ or more in area were identified in 42 eyes of 28 patients. The distribution of scar sizes in the fellow eye of patients with one scar measuring $3 \times 10^{6} \mu \mathrm{m}^{2}$ or more is charted in Figure 3. In $21.4 \%$ (6/28) the scar in the fellow eye measured less than $0.5 \times$ $10^{6} \mu^{2}$, and in $28.6 \%(8 / 28)$ measured less than $1 \times 10^{6} \mu \mathrm{m}^{2}$. In $50 \%(14 / 28)$ the fellow eye scar was 3 or more $\times 10^{6} \mu \mathrm{m}^{2}$ in area.

We compared the distribution of scar sizes in fellow eyes of eyes with small scars $\left(<0.5 \times 10^{6}\right.$ $\mu \mathrm{m}^{2}$ ) with that of fellow eyes of eyes with large scars $\left(>3.0 \times 10^{6} \mu \mathrm{m}^{2}\right)$. The data summarised in Figure 3 were grouped into three size distri-

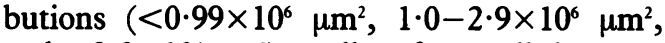
and $\left.>3.0 \times 10^{6} \mu \mathrm{m}^{2}\right)$ to allow for small data sets in the mid range of sizes. A significant difference in distribution between the groups was found

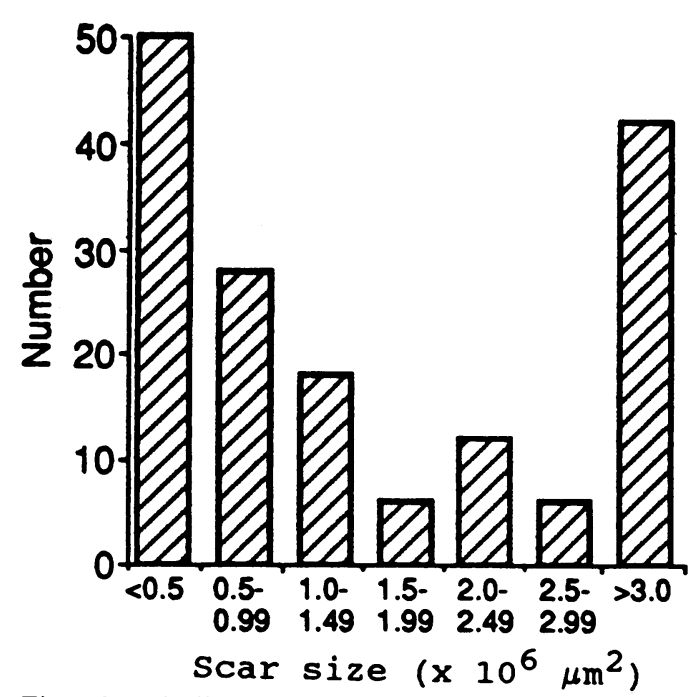

Figure 2 Distribution of macular scar sizes in all eyes.

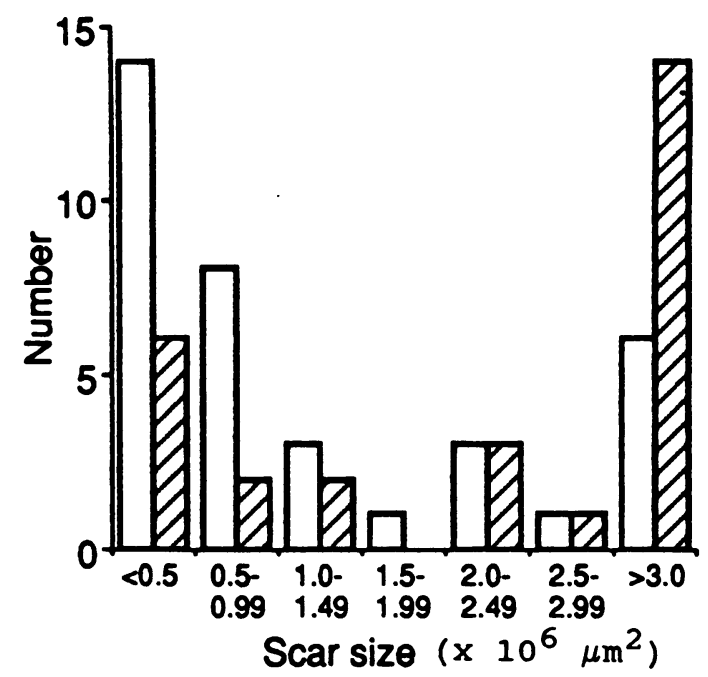

Figure 3 Fellow eyes of small scars compared with fellow eyes of large scars by scar size. $\left(\chi^{2}=20.07,=2, p<0.001\right)$. $\square=$ Fellow eye of small scar. $\square=$ Fellow eye of large scar.

with the $\chi^{2}$ test $\left(\chi^{2}=20 \cdot 07,2\right.$ degrees of freedom, $\mathrm{p}<0.001$ ).

Figure 4 demonstrates that a correlation exists between the scar size in one eye and the scar size in the fellow eye. Although a number of outliers exist, a clear uptrend is present $(r=0.53$, $\mathrm{p}<0.001 ; 95 \%$ confidence level for correlation coefficient: $0 \cdot 37-0 \cdot 77$ ).

We also assessed the degree of concordance of scar size using the $x$ statistic. ${ }^{15}$ This produced a weighted $x$ score of 0.547 (standard error $=$ $0 \cdot 153$ ), indicating a degree of concordance rated as 'fair to good'. ${ }^{16}$

We examined the scar sizes in 20 pairs of eyes with a minimum of six months' follow-up, and compared them with a group of 18 eyes with a minimum of three years' follow-up. There was no significant difference in the distribution of scar sizes between the two groups $(p=0 \cdot 36)$. We were concerned that eyes with a shorter duration of follow-up might have had evolving lesions which had not yet reached their final scar size. We re-examined the question of concordance of scar size for 43 patients with a minimum follow-

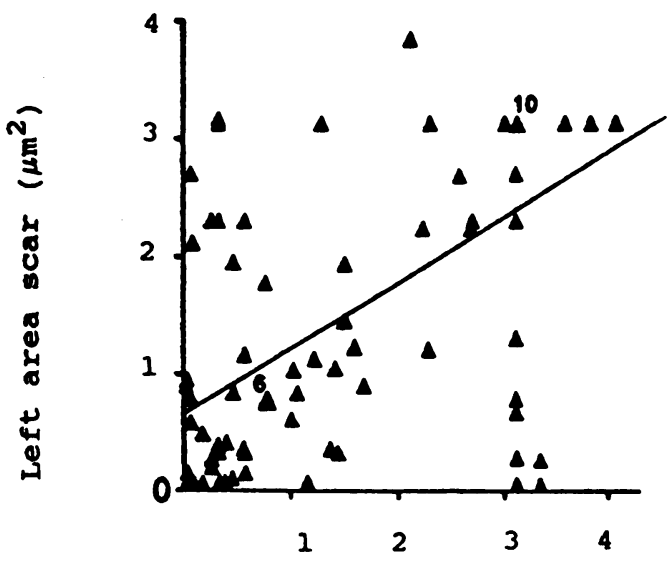

Right area scar $\left(\mu \mathrm{m}^{2}\right)$

Figure 4 Area of scar: right and left eyes of pairs compared. Numbers on chart refer to number of pairs of eyes located at similar points. Line of best fit has been added $(r=0.53$, $p<0.001 ; 95 \%$ confidence level for correlation coefficient: $0 \cdot 37-0 \cdot 77)$. 
up period of 12 months, and found a weighted $x$ score of 0.68 . The time of presentation did not appear to influence subsequent scar size - that is the first affected eye did not have a tendency towards larger or smaller scars.

We examined lesions for the presence of pronounced pigmentation, intraretinal exudate, and persistent subretinal fluid. We identified 17 eyes in which pigment was a major feature of the AMD lesion. In eight eyes $(47 \%)$ the macular scar size was less than $1.5 \times 10^{6} \mu \mathrm{m}^{2}$, while only $12 \cdot 7 \%$ eyes with no pigment epithelial hyperplasia had lesions of this size $(\mathrm{p}<0.001)$.

Circinate exudate was identified as a major feature of macular scars in 14 eyes, 12 of which $(85 \cdot 7 \%)$ had lesions measuring more than $3 \times 10^{\circ}$ $\mu \mathrm{m}^{2}$.

\section{Discussion}

We have found a relationship between the scar size of one eye and the size of the scar in the fellow eye of eyes with SRNVM secondary to AMD. This implies that factors peculiar to a particular patient have an important role in determining how advanced the process of subretinal neovascularisation and fibrosis may become. It has been noted that patients with AMD are significantly more likely to have a family history of central visual loss suggestive of AMD than controls. ${ }^{2}$ That genetic factors may contribute to the risk of AMD is suggested by the report of monozygotic twins with similar AMD lesions. ${ }^{17}$ This is supported by the finding that patients with AMD may have raised serum caeruloplasmin levels, and that this abnormality may be familial. ${ }^{18}$ The lower incidence of AMD in Asian $\mathbf{s}^{19}$ and blacks ${ }^{20}$ provides further evidence for the importance of inherited factors.

Systemic associations with AMD have been documented, and these include elastotic degeneration of the dermis, ${ }^{21}$ reduced hand grip strength, ${ }^{2}$ and cigarette smoking. ${ }^{2}$ It is possible that the similarity in scar sizes between eyes in the same individual may reflect the effect of some underlying inherited or systemic factor on the behaviour of SRNVM complicating AMD.

Alternatively factors peculiar to the eyes, but symmetrical, may influence scar size. A recent study has found a marked degree of concordance in the characteristics of drusen of eyes with AMD in the absence of SRNV. ${ }^{\star}$ While our study shows a degree of concordance, it is lower than that found by Barondes et al, ${ }^{\star}$ and it may reflect the multitude of factors involved in fibrovascular scar formation. Since drusen type determines the relative risk of SRNVM formation, ${ }^{22}{ }^{23}$ the high degree of symmetry of drusen type and extent found by Barondes $e t a l^{\star}$ may be a factor in the high frequency of bilaterality and the symmetry of SRNVM secondary to AMD.

We have noted that almost half the eyes (47\%) with pigmented scars have lesions measuring less than $1.5 \times 10^{6} \mu \mathrm{m}^{2}$. The retinal pigment epithelium plays a part in regulating the behaviour of SRNVM complexes, ${ }^{24}$ and it is possible that this could be a factor in determining the final scar size and the correlation between

^Barondes $\mathrm{MJ}$, et al unpublished eyes. It is not clear at what point in the evolution of a SRNVM this feature becomes evident, but this could have prognostic implications.

Most of the eyes with considerable exudation had large macular scars $(85 \cdot 7 \%)$, and this may reflect the activity of the underlying SRNVM, or its choroidoretinal vascular shunts. While a good degree of concordance of scar size between eyes was found for the study group as a whole $(x=$ 0.54 ), we found that the degree of concordance was increased when we considered only those patients with at least 12 months' follow-up $(x=$ 0.68 ). This implies that some of the variation between eyes may be attributed to lesions which are slowly evolving and have yet to reach their final scar size.

Our findings have implications for treatment of individual patients. It is not uncommon to encounter a patient with an established macular scar from SRNVM secondary to AMD in one eye, and a SRNVM within the foveal avascular zone in the fellow eye. In such patients treatment carries a significant risk of compromising foveal function. ${ }^{14}$ While SRNVMs with large vascular channels are thought to have slower growth, ${ }^{25}$ a recent study could not identify any morphological features indicative of rapid growth. ${ }^{26}$ Our results suggest that, in patients whose established macular scar is small, the risk of developing a large macular scar in the remaining eye is approximately $16 \%$, and aggressive treatment in these patients may not be justified. By contrast, in patients with a large established scar the risk to the paracentral visual function of the fellow eye is considerable, since 50\% develop large scars, and aggressive treatment in these cases could be considered.

A further clinical problem is that of the eye with a single large scar resulting from SRNVM secondary to AMD, and a fellow eye with AMD but no evidence of SRNVM formation. Previous studies have suggested that these eyes are exposed to a 7-15\% cumulative annual incidence involvement of the fellow eye with SRNVM. ${ }^{57}$ This implies that $30-50 \%$ of fellow eyes would have suffered SRNVM at five years. Since $50 \%$ of these eyes will have large scars, $25 \%$ of the original group are likely to develop bilateral severely incapacitating macular lesions. If one arbitrarily defines a large scar as any lesion larger than $2.0 \times 10^{6} \mu \mathrm{m}^{2}$, then the percentage rises to $32 \%$.

In a review of a sequential series of patients with SRNVM formation secondary to AMD, the majority had scar sizes larger than one disc diameter $(68 \%) .^{9}$ It is interesting to note a biphasic distribution of scar size in the study (Fig 2). This biphasic peak suggests that different factors determine whether an eye develops either a small or a large scar. Such an 'on or off' response is compatible with several types of cellular phenomena, and may be the end result of a retinal environment inhibitory to or stimulating SRNVM formation. In this light it is interesting to note that the retinal pigment epithelium may have a major role in regulating the activity of laser induced experimental SRNVMs ${ }^{2+}$ and of vascular endothelial cells. ${ }^{28}$

Some evidence suggests that inflammatory and immunocompetent cells may play a part in 
the genesis of both the atrophic forms of $\mathrm{AMD}^{29}$ and its neovascular complications. ${ }^{30}$ Immunological responses vary markedly between individuals, and can effect damping or recruitment - that is, 'on or off' responses. It is possible that factors such as these may explain in part the marked variation between individuals in final scar size, the close symmetry between eyes, and the bimodal distribution of scar sizes found in this study.

We considered whether our results could have been the result of biased case collection. We included all sequential patients who fulfilled the entry criteria, but the high mortality in this age group meant that a number of patients could not be included owing to inadequate follow-up. However, we found no obvious differences in the initial photographs of those patients with inadequate follow-up compared with those entered in this study. It is possible that the high peak in the group with large scars may represent patients with severe disease who attend frequently for visual aid; however, we found no difference in follow-up between the small scar and large scar groups.

In summary, we have found that eyes with a large macular scar resulting from age-related SRNVM formation have a high probability of developing a large scar if SRNVM forms in the remaining eye. This has implications for the management of patients and for the design of clinical trials.

We are grateful to Professor Alan Bird for his helpful comments and advice.

1 Ghafour M, Allan D, Foulds WS. Common causes of blindness and visual handicap in the West of Scotland. $\mathrm{Br} F$ Ophthalmol 1983; 67: 209-13.

2 Hyman LG, Lilienfeld AM, Ferris FL III, Fine SL. Senile macular degeneration: a case-control study. Am $\mathcal{f} E$ pidemio 1983; 118: 213-27.

3 Nasrallah FP, Jalkh AE, Friedman GR, Trempe CL, McMeel $\mathrm{JW}$, Schepens CL. Visual results with low-vision aids in age-
related macular degeneration. Am $\mathcal{F}$ Ophthalmol 1988; 106: related

4 Weiter JJ, Wing GL, Trempe CL, Mainster MA. Visual acuity related to retinal distance from the fovea in macular disease. Ann Ophthalmol 1984; 16: 174-6.

5 Gregor Z, Bird AC, Chisholm IH. Senile disciform macula degeneration in the second eye. Br $₹$ Ophthalmol 1977; 61 : $141-47$.

6 Leibowitz H, Krueger DE, Maunder LR. The Framingham Eye Study Monograph: an ophthalmological and epidemiological study of cataract, glaucoma, diabetic retinopathy, macular degeneration. Surv Ophthalmol 1980; 24: 335-610.
7 Coffey AJH, Brownstein S. The prevalence of macular drusen in postmortem eyes. Am F Ophthalmol 1986; 102: 164-71.

8 Chuang EL, Bird AC. Bilaterality of tears of the retinal pigment epithelium. Br f Ophthalmol 1988; 72: 918-20.

9 Berkow JW. Subretinal neovascularization in senile macular degeneration. Am f Ophthalmol 1984; 97: 143-7.

10 Grey RHB, Bird AC, Chisholm IH. Senile disciform macular degeneration: features indicating suitability for photocoagulation. Br f Ophthalmol 1979; 63: 85-9.

11 Folk JC. Aging macular degeneration: clinical features of treatable disease. Ophthalmology 1985; 92: 594-602.

12 Bressler SB, Bressler NM, Fine SL, McCormick P, Auer C. Subfoveal neovascular membranes in senile macular degeneration: relationship between membrane size and visual prognosis. Retina 1983; 3: 7-11.

13 Guyer DR, Fine SL, Maguire MG, Hawkins BS, Owens SL Murphy RP. Subfoveal choroidal neovascular membranes in age-related macular degeneration: visual prognosis in eyes age-related macular degeneration: visual prognosis in eyes
with relatively good initial visual acuity. Arch Ophthalmol with relatively good

14 Melrose MA, Magargal LE, Donoso LA, Goldberg RE, Edmonds SE. Vision parameters in krypton and laser photocoagulation of subfoveal neovascular membranes. Ophthalmic Sür 1985; 16: 495-502.

15 Fleiss JL. Statistical methods for rates and proportions. 2nd ed. New York: Wiley, 1981.

16 Landis RJ, Koch GG. The measurement for observer agreement for categoric data. Biometrics $1977 ; 33: 159-74$.

17 Melrose MA, Magargal LE, Lucier AC. Identical twins with subretinal neovascularization in complicating senile macular subretinal neovascularization in complicating senil

18 Newsome DA, Swartz M, Leone NC, Hewitt AT, Wolford F Miller ED. Macular degeneration and elevated serum Miller ED. Macular degeneration and elevated serum

19 Hoshino M, Mizuno K, Ichikawa H. Aging alterations of retina and choroid of Japanese: light microscopic study of macular region of 176 eyes. $\mathcal{F}$ pn $\mathcal{F}$ Ophthalmol 1984; 28: 89102

20 Gregor ZJ, Joffe L. Senile macular changes in the black African. Brf Ophthalmol 1978; 62: 547-50.

21 Blumenkranz MS, Russell SR, Robey MG, Kott-Blumenkranz $\mathbf{R}$, Penneys N. Risk factors in age related maculopathy complicated by choroidal neovascularization. Ophthalmology 1986; 93: 552-7.

22 Bressler NM, Bressler SB, Gragoudas ES. Clinical characteristics of drusen in patients with exudative versus nonexudative age-related macular degeneration. Retina $1988 ; 8$ : exudative 14.

23 Smiddy WE, Fine SL. Prognosis of patients with bilateral macular drusen. Ophthalmology 1984; 91: 271-7.

24 Miller H, Miller B, Ryan SJ. The role of retinal pigmen epithelium in the involution of subretinal neovascularization. Invest Ophthalmol Vis Sci 1986; 27: 1644-52.

25 Teeters VW, Bird AC. The development of neovascularization of senile disciform macular degeneration. Am $\mathcal{F}$ Ophthalmo 1973; 76: 1-18

26 Klein ML, Jorizzo PA, Watzke RC. Growth features of choroidal neovascular membranes in age-related macular degeneration. Ophthalmology 1989; 96: 1416-21.

27 Strahlman ER, Fine SL, Hillis A. The second eye of patients with senile macular degeneration. Arch Ophthalmol 1983; with senile

28 Glaser BM, Campochiaro PA, Davis JL Jr, Jerdan JA. Retinal pigment epithelial cells release inhibitors of neovascularization. Ophthalmology 1987; 94: 780-4.

29 Penfold PL, Killingsworth MC, Sarks SH. Senile macular degeneration. The involvement of giant cells in atrophy of the retinal pigment epithelium. Invest Ophthalmol Vis $S \mathrm{C}$ 1986; 27: 364-71.

30 Penfold PL, Provis JM, Billson FA. Age-related macular degeneration: ultrastructural studies of the relationship of leucocytes to angiogenesis. Graefes Arch Clin Exp Ophthalmol 1987; 225: 70-6. 